\title{
Epidemia de influenza del año 2009 en un hospital pediátrico y costos médicos directos en menores de 5 años comparados con el período 2006-2008 Impact on the direct medical cost related to the influenza virus during 2009 in children under 5 years compared to the period 2006-2008 in a pediatric hospital
}

\author{
Dr. Norberto D. Giglio ${ }^{a}$ Dra. Vanesa E. Castellano ${ }^{a}$, Dr. Ricardo W. Rüttimann ${ }^{b}$, \\ Dra. Gabriela I. Vidalc y Dra. Ángela Gentile
}

\section{RESUMEN}

Introducción. Anualmente se producen brotes epidémicos de variada intensidad atribuibles a Influenza y las instituciones de salud multiplican su capacidad de respuesta en términos de recursos médicos. Este cuadro genera un incremento en los costos institucionales durante la circulación del virus y, con la aparición del nuevo virus pandémico Influenza A (pH1N1), los costos podrían haber aumentado en relación a los años anteriores.

Objetivo. Valorar los costos directos en salud atribuibles a influenza durante el año 2009 y compararlo con años previos en el Hospital de Niños Ricardo Gutiérrez (HNRG) de la Ciudad de Buenos Aires, Argentina.

Material y métodos. Con datos de las consultas ambulatorias einternaciones por Infección Respiratoria Aguda atribuibles a Influenza de los años

a. Servicio de Promoción y Protección de la Salud. Área Epidemiología. Hospital de Niños Ricardo Gutiérrez.

b. Fundación Centro de Estudios Infectológicos. c. Novartis Vacunas Argentina.

Ciudad Autónoma de Buenos Aires.

Correspondencia:

Dr. Norberto D. Giglio: norbergiglio@gmail.com

Conflicto de intereses: El estudio se realizó con un subsidio irrestricto otorgado por Novartis Argentina y la Dra. Gabriela Vidal se desempeña como Gerente de Asuntos Médicos-Latinoamérica Sur en dicha compañía.

Recibido: 8-8-2011 Aceptado: 19-12-2011
2006 a 2009 se realizó un estudio epidemiológico de corte transversal, con control retrospectivo, para valorar costos en niños menores de 5 años. Con los costos obtenidos para cada año, se realizó el cálculo del costo incremental del período de estudio 2009 en relación al costo promedio de los períodos 2006-2008.

Resultados. El costo incremental total (hospitalizaciones y consultas ambulatorias por influenza) del período de estudio 2009 en relación al costo promedio del período 2006-2007-2008 fue US\$ 91512 . Para las hospitalizaciones, el costo incremental del período de estudio 2009 en relación al costo promedio del período 2006-2007-2008 fue de US $\$ 147560$ y de la consulta ambulatoria de US\$ -56 048 .

Conclusiones. El período de estudio 2009 generó un costo mayor en relación al promedio de los años 2006-2007-2008, asociado al número de hospitalizaciones atribuibles a Influenza A (pH1N1). Palabras clave: subtipo H1N1 del virus de la Influenza $A$, costos de la atención en salud.

\section{SUMMARY}

Introduction. Epidemic outbreaks of influenza occur every year and healthcare systems must amplify their response in accordance with available medical resources. This situation generates cost increases. During the recent influenza A pandemic, costs were likely to have been greater than in previous years.

Objective. To estimate direct health costs of in- fluenza-related illness during 2009 compared to costs expended in the previous 3-year period at Ricardo Gutiérrez Children's Hospital, City of Buenos Aires, Argentina.

Materials and methods. We conducted a crosssectional epidemiological study with retrospective control group to estimate influenza-related costs for the years 2006 to 2009 using data from outpatient consultations and hospital admissions for Acute Respiratory Infections attributable to Influenza virus in children under 5 years old. Incremental costs were estimated for the year 2009 compared to average costs for the period 2006-2008.

Results. Total incremental cost of hospital admissions and outpatient consultations for influenza was US\$91512 for the year 2009 compared to average cost for 2006-2008 period (incremental cost of US\$ 147560 for hospital admissions and US\$ -56048 for outpatient consultations).

Conclusions. Influenza-related costs were greater in 2009 than in previous years due to higher number of hospital admissions attributable to influenza A H1N1.

Key words: Influenza A virus H1N1 subtype, health care costs.

http:/ /dx.doi.org/10.5546/aap.2012.19

\section{INTRODUCCIÓN}

El 11 de junio de 2009 la Organización Mundial de la Salud (OMS) declaró oficialmente el estado de pandemia debido a la diseminación de un nuevo virus influenza A ( $\mathrm{pH} 1 \mathrm{~N} 1)$ en diversos lugares del mundo. ${ }^{1}$

La Argentina fue uno de los países más afectados por este virus, situación que generó un impacto sustancial sobre la capacidad operativa de los sistemas locales de salud.

Si bien anualmente se producen brotes epidémicos de variada intensidad asociados a infecciones respiratorias agudas (IRA) y las instituciones 
de salud multiplican su capacidad de respuesta, el aumento de la tasa de ataque generado por la pandemia, junto a la incertidumbre en la evolución clínica de los pacientes frente a este nuevo virus, reforzó nuestra hipótesis de un posible aumento en los costos durante el período de circulación de influenza A (pH1N1) en relación a años previos. ${ }^{2,3}$

El objetivo de este estudio fue valorar los costos directos en salud atribuibles a influenza durante el año 2009 y compararlo con años previos en el Hospital de Niños Ricardo Gutiérrez (HNRG) de la Ciudad Autónoma de Buenos Aires, Argentina.

\section{MATERIAL Y MÉTODOS}

Diseño: estudio de corte transversal, con control retrospectivo, para valorar costos atribuibles a influenza durante el período de circulación del virus influenza del año 2009 comparado con el promedio de los años 2006, 2007 y 2008.

Mediante la distribución de los patrones de circulación viral informados por el Programa de Vigilancia Epidemiológica de IRA baja (IRAB) del HNRG ${ }^{4,5}$ se definió el período de cuantificación de casos atribuibles a influenza 2009; 2008; 2007; 2006 de acuerdo a los lineamientos establecidos por Izurieta y cols., ${ }^{6}$ para el período de circulación extendida donde se define la existencia de casos atribuibles a influenza, cuando se produce un período de dos o más semanas consecutivas en el año, en el cual para cada semana se registra al menos un 5\% de aislamiento de influenza del total de la temporada (ver Gráfico 1).

De acuerdo al diseño descripto se realizó un macrocosteo con el propósito de cuantificar y asignar costos a: número de hospitalizaciones, días de internación en la sala de clínica médica y la Unidad de Terapia Intensiva Pediátrica (UTIP); y número de consultas por IRA y radiografías de tórax solicitadas.

Para el período definido previamente fueron seleccionados todos los niños menores de 5 años, con factores de riesgo y sin ellos, que se hospitalizaron según los códigos CIE107 J10, J11, J12.9, J13, J15, J15.8, J15.9, J18, J20 y J21 con diagnóstico virológico positivo por la técnica de inmunofluorescencia indirecta (IFI) para influenza. Debido a que existe un porcentaje de pacientes con diagnóstico falso-negativo por la técnica de IFI y teniendo en cuenta el uso de control retrospectivo, donde la práctica de esta técnica podría haber variado, se decidió incluir además, para todos los años de estudio, las IRAB con IFI negativa o no realizada. Finalmente y a los fines de acotar el sesgo de circulación concomitante de otros virus se excluyeron los casos positivos para virus respiratorio sincicial (VRS).

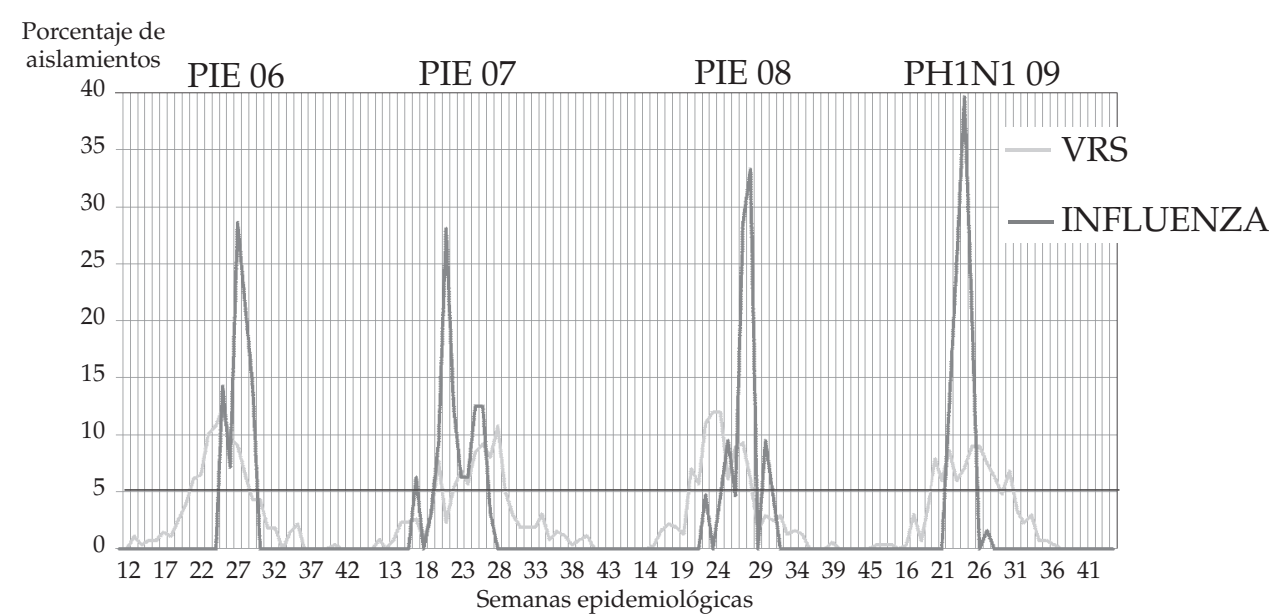

Fuente: Programa de Vigilancia Epidemiológica de IRA baja del HNRG. Años 2006-2009.

VRS: Virus respiratorio sincicial.

PIE 06: Período de influenza estacional, año 2006.

PIE 07: Período de influenza estacional, año 2007.

PIE 08: Período de influenza estacional, año 2008

PIH1N1 09: Período de influenza A (pPH1N1), año 2009. 
Para los mismos periodos de circulación de virus de influenza se estimó el porcentaje de consultas ambulatorias por IRA (CIE107 J00 a J04, J06, J10, J 11 J12.9, J13, J15, J15.8, J15.9, J18, J20 y J21) en relación al total de consultas registradas. Para la estimación del porcentaje se seleccionó una muestra de 8555 consultas del total registradas $(23010)$ durante los PIE 06; 07; 08 y PIH1N1 de los servicios de atención ambulatoria y el servicio de urgencias en la Institución.

Los precios unitarios de los recursos en salud (día de internación, consulta ambulatoria y radiografía de tórax) se ajustaron a un valor común en pesos para todos los períodos de acuerdo al valor asignado por el Nomenclador de Prestaciones de Salud del Gobierno de la Ciudad de Buenos Aires, primer semestre de $2010^{8}$ y se convirtieron al valor dólar, asumiendo un tipo de cambio de US\$ $1=\$ 3,98$.

Para el cálculo del costo total (hospitalizaciones y consultas) de cada período anual se excluyeron costos a largo plazo, como, por ejemplo, aquellos originados por las secuelas de los casos agudos.
Con los datos del costo total, para cada año, se calculó el costo incremental ${ }^{\mathrm{b}}$ y exceso atribuible ${ }^{\mathrm{c}}$ de utilización de recursos del Período de Influenza A (pH1N1) (PIH1N1) comparado con el Período de Influenza estacional de los años 2006, 2007 y 2008 (PIE 06-08) d. El análisis se realizó en forma global y parcial, estratificado por internación y consultas ambulatorias.

A los fines del tratamiento de la incertidumbre se realizó un análisis de sensibilidad univariado donde se modificaron en el modelo inicial las hospitalizaciones que solo presentaron IFI positiva para influenza y el porcentaje de consulta ambulatoria por IRA, de acuerdo al intervalo de confianza (IC) máximo y mínimo del 95\%, obtenido mediante la técnica de muestreo.

Finalmente, se exploró un escenario parcial donde solo se compararon las hospitalizaciones con IFI positiva en los períodos de estudio.

Para la estimación de los IC se utilizó el programa estadístico Epi Info, versión 6.04 y Open Epi, versión 2.3.1. ${ }^{9}$

GRÁfICO 2. Distribución de los días de hospitalización por infección respiratoria aguda durante los períodos de estudio según frecuencia

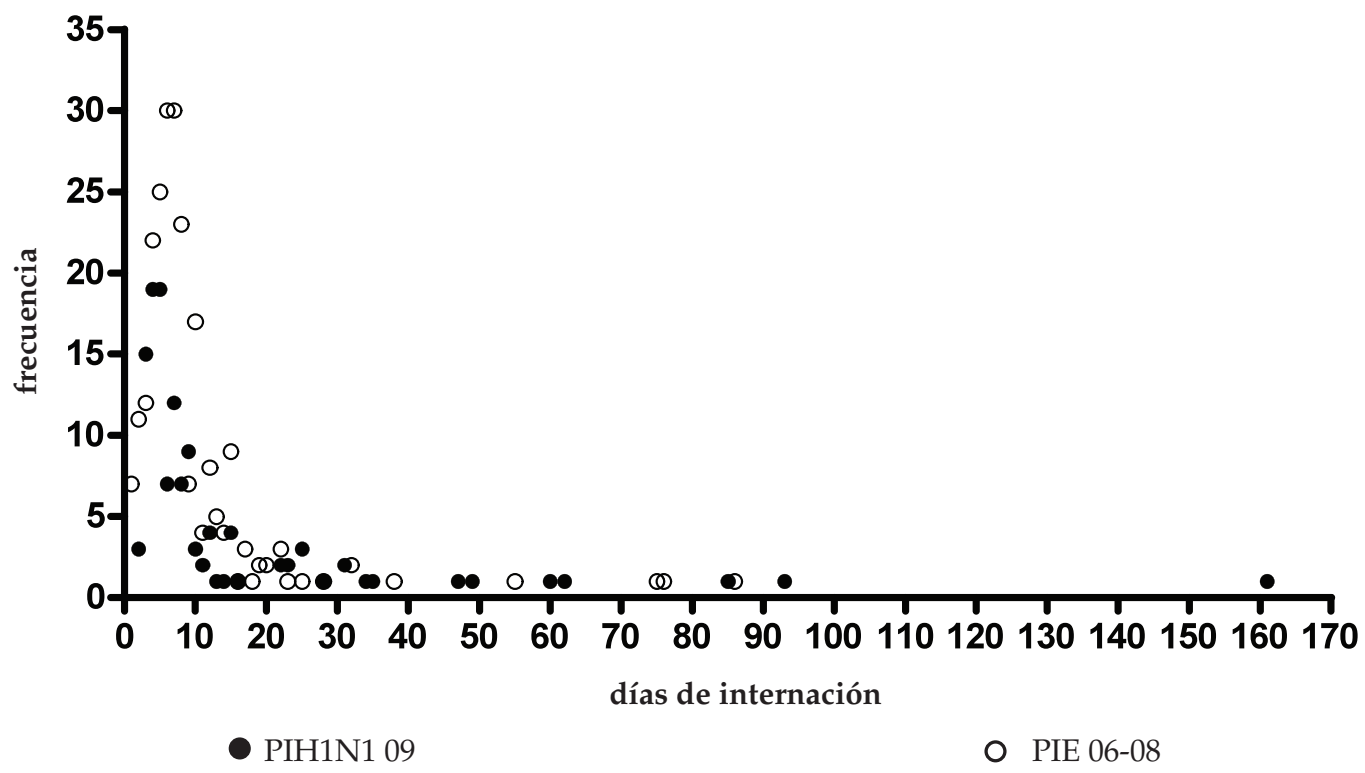

PIE 06-08 Período de influenza estacional, años 2006-2008.

PIH1N1 09: Período de influenza A (pPH1N1), año 2009.

a. En él están incluidos los costos de análisis de laboratorio generales, radiografía de tórax, antibióticos betalactámicos de primera generación, oxígeno, kinesiología respiratoria y material descartable.

b. Costo incremental= costo del PIH1N1 - Costo del PIE 06-08.

c. Exceso atribuible a influenza A (pH1N1): número de internaciones o consultas durante el PIH1N1 menos el número de internaciones o consultas en el PIE 06-08.

d. El período 2006-2008 (PIE 06-08) se calculó como el resultado promedio del costo de los años 2006, 2007 y 2008. 


\section{RESULTADOS}

El Período de circulación de Influenza A ( $p H 1 N 1)$ estuvo comprendido entre el 7 de junio y el 4 de julio de 2009, correspondiendo a las semanas epidemiológicas 23-26 (ver Gráfico 2).

Doce pacientes, que corresponden al 9,37\% (IC: 5,17-15,39) de los pacientes hospitalizados, requirieron internación en UTIP. De la población hospitalizada durante este período, el 59\% fueron varones y el $57 \%$ presentó factores de riesgo de complicación con el virus influenza.

El Período de circulación de Influenza estacional 06-08 estuvo comprendido entre el 25 de junio y el 5 de agosto de 2006, el 20 de mayo al 7 de julio de 2007 y del 6 de julio al 19 de julio de 2008, correspondiendo a las semanas epidemiológicas 26-31 para el 2006, 21-27 para el 2007, 28-29 para el 2008 (ver Gráfico 2). Doce pacientes, que corresponden al 5\% (IC: 2,73 -8,34) de los pacientes hospitalizados, requirieron internación en UTIP, siendo el promedio anual de 4 pacientes.

Durante ese período, el 53,3\% de los pacientes hospitalizados fueron varones y el 59,1\% presentó factores de riesgo.

Del total de las consultas ambulatorias, la proporción de visitas en guardia (urgencias) fueron en el PIE 06 41,8\%, PIE 07 45,61\%, PIE 08 42,54\% y PIH1N1 48,18\%.
En la Tabla 1 se describen los recursos en salud cuantificados en cada uno de los períodos.

En la Tabla 2 se describen los costos de cada período y en el Gráfico 3 distribución porcentual.

El costo incremental total del PIH1N1 en relación al promedio anual del PIE 06-08 fue de US\$ 91 512. En relación a las hospitalizaciones y consultas ambulatorias, el costo incremental (PI H1N1 - PIE 06-08) fue de US\$ 147560 y US\$ -56 048 respectivamente.

El exceso atribuible al PIH1N1 de recursos utilizados comparado con el promedio anual del PIE 06-08 fue de 50 internaciones y 591 días de internación en sala de Clínica Médica, 8 internaciones y 289 días de internación en UTIP, y existió un balance negativo para el número de consultas ambulatorias (-2378) y radiografías de tórax solicitadas (-455).

En el análisis de sensibilidad, cuando se incorporaron al modelo solamente las hospitalizaciones con IFI positiva para influenza junto al total de las consultas ambulatorias por IRA, el costo fue de US\$ 160482 en el PIH1N1 de US\$ 175 814/ año en el PIE 06-08. Siendo el costo incremental total: US\$ 15332.

Cuando se consideraron los IC de 95\% para la estimación del número de consultas por IRA, el costo incremental total del PH1N1 en rela-

TABLA 1. Utilización de recursos en niños menores de 5 años durante los períodos de estudio

\begin{tabular}{|c|c|c|c|c|c|c|}
\hline Períodos & & PIH1N1 & PIE 06-08 & PIE 08 & PIE 07 & PIE 06 \\
\hline \multicolumn{7}{|c|}{ Clínica médica } \\
\hline $\mathrm{N}^{\mathrm{o}}$ internaciones & & 128 & 80 & 34 & 123 & 77 \\
\hline $\begin{array}{l}\text { Media y mediana de } \\
\text { días de internación }\end{array}$ & & 4-6,5 (IQR: 4-11) & 9,1-6,6 (IQR: 4-11) & 12,7-7 (IQR: 4-15) & 8,9-7 (IQR: 5-10) & 7,9-6 (IQR: 4-88) \\
\hline Días totales de interna & ción $^{1}$ & 1213 & 622 & 399 & 927 & 539 \\
\hline \multicolumn{7}{|c|}{ UTIP } \\
\hline $\mathrm{N}^{o}$ de internaciones & & 12 & 4 & 5 & 3 & 4 \\
\hline $\begin{array}{l}\text { Media y mediana de } \\
\text { días de internación }\end{array}$ & 23,6 & 6-22,5 (IQR: 12-34) & 16,5-17,8 (IQR: 7,6-35,6) & 9,8-7,5 (IQR: 5-14) & 23-27 (IQR: 8-56) & 19,2-19 (IQR: 10-37) \\
\hline $\begin{array}{l}\text { Días totales de } \\
\text { internación en UTIP² }\end{array}$ & & 354 & 65 & 38 & 91 & 66 \\
\hline \multicolumn{7}{|c|}{ Atención ambulatoria } \\
\hline $\begin{array}{l}N^{\circ} \text { total de consultas } \\
\text { ambulatorias }\end{array}$ & & 8230 & 10608 & 3883 & 15108 & 12832 \\
\hline $\begin{array}{l}\mathrm{N}^{\circ} \text { total de radiografia } \\
\text { de tórax ambulatorias }\end{array}$ & & 3505 & 3960 & 1545 & 6347 & 3988 \\
\hline
\end{tabular}

PIE 06: Período de influenza estacional, año 2006.

PIE 07: Período de influenza estacional, año 2007.

PIE 08: Período de influenza estacional, año 2008.

PIE 06-08: Promedios período de influenza estacional, años 2006, 2007 y 2008.

PIH1N1: Período de influenza A (pPH1N1), año 2009.

IQR: Intervalo intercuartil. 
ción al PIE 06-08 varió entre US\$ 93295 y US\$ 89762 para el máximo y mínimo de las consultas esperadas.

En el escenario parcial, cuando se analizaron sólo las hospitalizaciones con IFI positiva para influenza, el costo fue de US\$ 59465 en el PIH1N1 y de US\$ 18749 (el costo promedio anual) durante el PIE 06-08, siendo el costo incremental de US\$ 40716.

\section{DISCUSIÓN}

De acuerdo a nuestros resultados, el mayor aumento en el costo del PIH1N1 se relacionó con el número de hospitalizaciones en Clínica Médica y pacientes internados en UTIP.

Las hospitalizaciones en Clínica Médica constituyen un exceso de 50 hospitalizaciones en relación al promedio de los años previos estudiados. Este exceso podría justificarse por factores como una mayor tasa de ataque de la infección, la saturación del sistema de salud que impide las deri-

TABLA 2. Costos de cada período en dólares

\begin{tabular}{|c|c|c|c|c|}
\hline Períodos & Internación de Clínica ${ }^{1}$ & Internación de UTIP ${ }^{2}$ & Consulta ambulatoria ${ }^{3,4}$ & TOTAL \\
\hline PIH1N1 & US\$ 146291 & US\$ 93392 & US\$ 101017 & US\$ 340700 \\
\hline PIE 06-08 Costo anual & US\$ 74 975/año & US\$ 17 148/año & US\$ 157 065/año & US\$ 249 188/año \\
\hline PIE 08 & US\$ 48121 & US\$ 10025 & US\$ 58867 & US\$ 117013 \\
\hline PIE 07 & US\$ 111799 & US\$ 24008 & US\$ 238092 & US\$ 373899 \\
\hline PIE 06 & US\$ 65005 & US\$ 17412 & US\$ 174235 & US\$ 256652 \\
\hline
\end{tabular}

*Fuente de los costos: Nomenclador de Prestaciones de Salud del Gobierno de la Ciudad de Buenos Aires. Primer semestre de 2010. Convertibilidad US $\$ 1=\$ 3.98$.

1- Costo por día de internación * US\$120.6.

2- Costo por día de internación en UTIP US\$ 263.8.

3- Costo por consulta Ambulatoria/Guardia US\$ 5.3/12.

4- Costo por radiografía de tórax ambulatoria US\$ 9.

PIE 06: Período de influenza estacional, año 2006.

PIE 07: Período de influenza estacional, año 2007.

PIE 08: Período de influenza estacional, año 2008.

PIH1N1: Período de influenza A (pPH1N1), año 2009.

$\%$

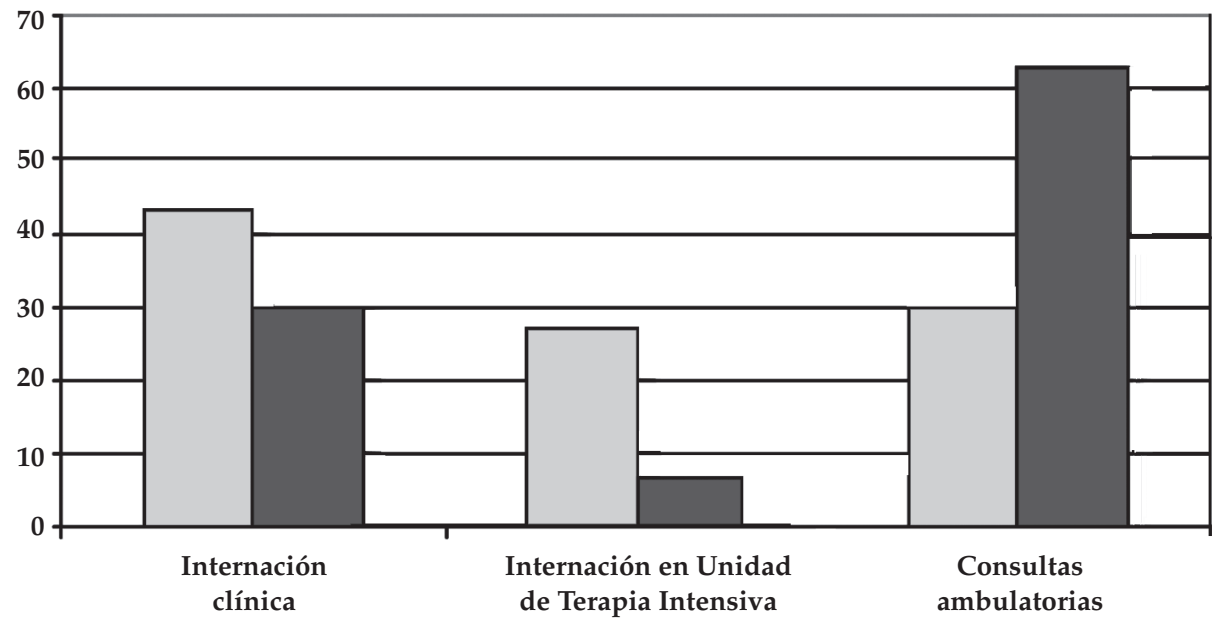


vaciones, la incertidumbre de los profesionales de la salud sobre la evolución clínica del nuevo virus pandémico, así como el impacto mediático y el temor de la población a repetir experiencias como la del SARS, la gripe aviar o la pandemia de $1918 .{ }^{10-13}$

Resulta interesante destacar que la mediana de días de hospitalización y la distribución del tiempo de estadía en internación según frecuencia (ver Gráfico 2) en sala de Clínica Médica del PIH1N1 fue semejante a la del PIE 06-08.

La mediana de hospitalización tanto para influenza estacional como pandémica ${ }^{5}$ es mayor a la descripta en otros estudios, ${ }^{14-17}$ donde para estos últimos, la mediana de internación oscila en 2-5 días. Condiciones como las características de los pacientes de mayor gravedad que concurren a un hospital de alta complejidad y la situación socioeconómica de nuestra población hospitalaria, podrían explicar estas diferencias con los trabajos publicados en países con mayor nivel de desarrollo socioeconómico.

En relación al porcentaje de pacientes internados en UTIP, si bien la proporción de ellos que requirió internación fue casi el doble en el PIH1N1, lo que permite suponer un mayor número de casos complicados, los intervalos de confianza del 95\% de ambas medias se superponen.

A diferencia de lo observado en las hospitalizaciones, las consultas ambulatorias por IRA mostraron un balance negativo en términos de costos.
Es probable que los mensajes de los medios de comunicación y las autoridades de Salud de "no concurrir a hospitales salvo bajo condición de gravedad", hayan acompañado este fenómeno, ${ }^{18,19}$ ya que durante el período invernal 2009, donde inicialmente el número de consultas a los servicios de salud generó una saturación del sistema, ${ }^{20} \mathrm{su}$ número descendió luego de las recomendaciones gubernamentales (ver Gráfico 4).

Nuestro trabajo destaca, además, que cada 2,5-3 pacientes que consultan por IRA se realiza una radiografía de tórax. Es por ello que, como lo demuestran otros trabajos, resulta posible que el número de radiografías por paciente que consulta sea elevado, ya que en los Servicios de Urgencia se suelen sobreestimar la probabilidad de neumonía de los enfermos que consultan por síntomas respiratorios, situación que deberá revalorarse en futuros estudios. ${ }^{21,22}$

El presente estudio también permitió observar que los costos de hospitalización del PIH1N1 fueron 2,6 veces mayores en relación al costo promedio anual del PIE06-08, principalmente asociados al número de hospitalizaciones.

En el análisis de escenario parcial, cuando solo se incluyeron pacientes hospitalizados con IFI positiva, el costo del PIH1N1 fue 3,17 veces mayor. Este resultado podría deberse a los patrones de comportamientos diferentes del virus influenza A (pH1N1), o a una evaluación diagnóstica más

Gráfico 4. Patrón de consulta semanal en el Departamento de Urgencias. Año 2009. Meses de mayo-agosto

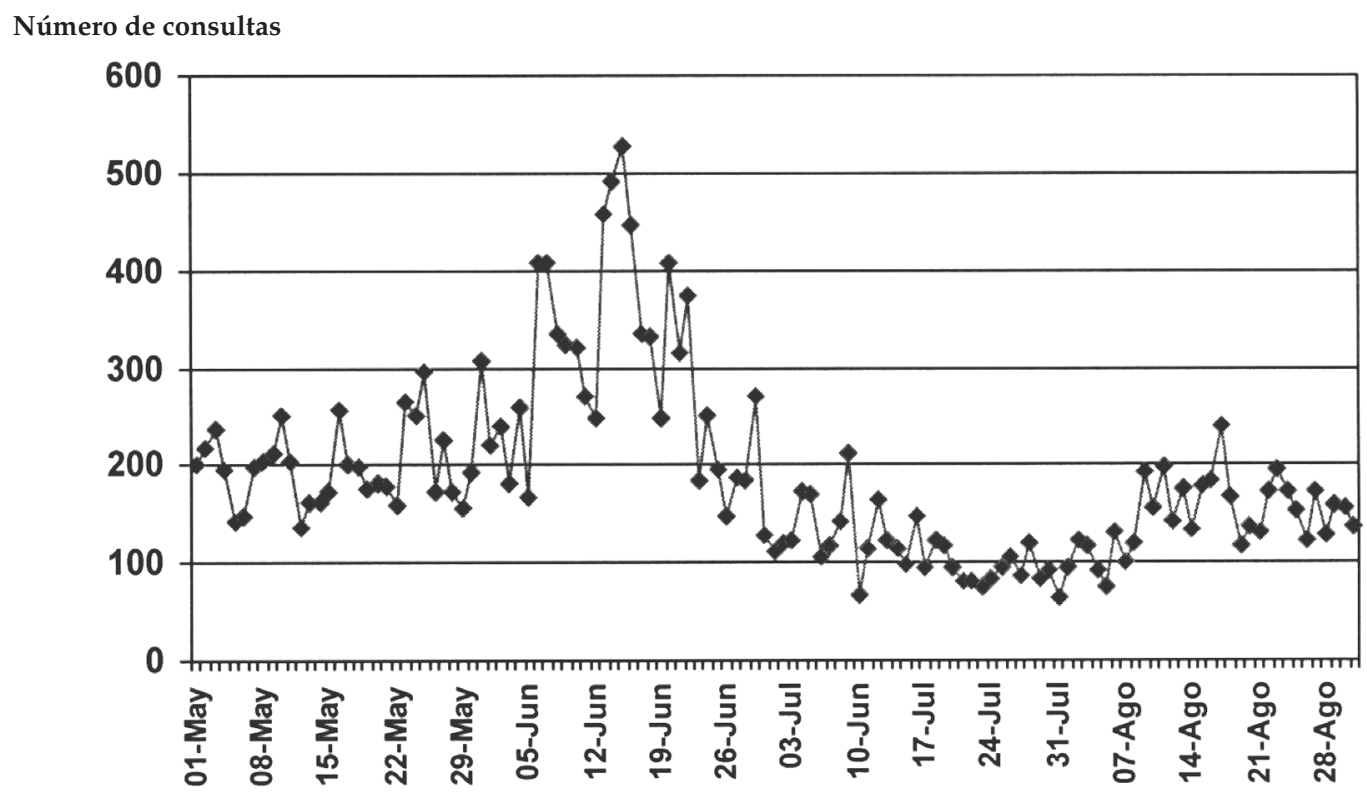

Fuente: Departamento de urgencias. Informe de consultas. Año 2009. 
precisa en la toma de la muestra de secreciones nasofaríngeas durante el año 2009, que podría haber modificado ${ }^{23}$ el valor predictivo positivo de la prueba en relación a las IRA con IFI negativas.

En relación al número de muestras no enviadas es posible que, durante el año 2009, el número de casos a los que se les solicitó muestra de IFI para secreciones nasofaríngeas haya sido mayor.

El balance negativo observado del costo incremental total en el análisis de sensibilidad cuando solo se consideraron hospitalizaciones con IFI positivo junto con las consultas por IRA se debe al mayor número de visitas ambulatorias en el PI0608 comparado con el PH1N1.

Esta modalidad de análisis epidemiológico donde se realiza una inferencia de casos atribuibles a influenza con control retrospectivo no está libre de sesgos. La circulación concomitante de otros virus respiratorios durante el período de estudio podría influir parcialmente en la valoración del impacto de la influenza A (pH1N1).

En el análisis estratificado de los períodos por año se observa que el costo total del PIE 07 supera al costo del PIH1N1, principalmente por el aumento de consultas ambulatorias. Contrariamente a lo observado en el PIE 08, de menor duración, el número de consultas ambulatorias resultó ser extremadamente bajo en relación a los PIE 07 y PIE 06, generando menores costos. Es por ello que el promedio de consultas y hospitalizaciones de al menos tres años, permite estimar un comportamiento basal, pero que podría modificarse si el control retrospectivo incluyera un número mayor de años estudiados debido a diferentes modalidades de circulación del virus influenza.

$\mathrm{Al}$ igual que los trabajos de valoración económica realizados con la vacuna antigripal estacional, ${ }^{24-26}$ el uso de esta intervención en los niños menores de cinco años podría reducir el número de consultas, hospitalizaciones y casos graves, $y$, evidentemente, influir en los costos asociados.

El Ministerio de Salud de la Nación Argentina ha incorporado desde el año 2010 la vacunación en niños de 6 meses a dos años, por lo que los resultados de este estudio podrán contribuir a la valoración del costo-eficacia de la implementación del programa.

\section{CONCLUSIONES}

El Período de Influenza A ( $p H 1 N 1)$ generó un costo mayor en relación al Período de Influenza estacional-06-08 y el mayor costo del PIH1N1 estuvo asociado al número de hospitalizaciones y no a la mediana de tiempo de hospitalización por pa- ciente en Clínica Médica, que fue similar en todos los períodos estudiados. El porcentaje de pacientes internados en UTIP durante el PIH1N1 sugiere una tendencia hacia un mayor riesgo de casos graves en relación a los períodos anteriores. A diferencia de lo observado en las hospitalizaciones, las consultas ambulatorias por IRA mostraron un balance negativo en términos de costos en relación al PIE 06-08.

\section{Agradecimientos}

A la Dra. Liliana Rabinovich, Jefa del Departamento de Estadística, quien nos facilitó los registros de las consultas ambulatorias.

Al Dr. Jorge Fiorentino, Jefe del Departamento de Urgencias, quien facilitó los registros médicos del departamento.

A la Dra. Liliana Sabbaj, Jefa del Servicio de Consultorios Externos, quien nos facilitó los registros médicos del Servicio.

Al Dr. Fernando Gentile, Jefe del Departamento de Imágenes, quien nos facilitó los registros de las radiografías de tórax realizadas en forma ambulatoria.

A la Dra. María Florencia Lución y Viviana Romanin, responsables del Programa de Vigilancia Epidemiológica de IRAB del hospital.

\section{Anexo I. \\ Códigos CIE10}

J00 Nasofaringitis aguda.

J01 Sinusitis aguda.

J02 Faringitis aguda.

J03 Tonsilitis aguda.

J04 Larinbgitis y traqueítis agudas.

J06 Infecciones agudas respiratorias superiores de sitios múltiples y sin especificar.

J10 Gripe debido a virus identificado de la gripe.

J11 Gripe, virus no identificado.

J12.9 Neumonía vírica sin otra especificación.

J13 Neumonía debida a Streptococcus pneumoniae.

J14 Neumonía debida a Haemophilus influenzae.

J15 Neumonía bacteriana, no clasificada en otra parte.

J15.8 Otras neumonías bacterianas.

J15.9 Neumonía bacteriana sin especificar.

J18 Neumonía, organismo sin especificar.

J20 Bronquitis aguda.

J21 Bronquiolitis aguda. 


\section{BIBLIOGRAFÍA}

1. Chan M. World now at the start of 2009 influenza pandemic. [Acceso: 24 de febrero de 2011]. Disponible en: http:/ / www.who.int/mediacentre/news/statements / 2009/ h1n1_pandemic_phase6_20090611/en/.

2. Writing Committee of the WHO Consultation on Clinical Aspects of Pandemic (H1N1) 2009 Influenza, Bautista E, Gao Z, Harper SA, Shaw M, et al. Clinical Aspects of Pandemic 2009 Influenza A (H1N1) Virus Infection. Review Article. N Engl J Med 2010; 362(18):1708-19.

3. Ministerio de Salud. Presidencia de la Nación. Vigilancia de Infecciones respiratorias Agudas en Argentina. Informe semanal 26/08/2010. [Acceso: 19 de diciembre de 2011]. Disponobleen:http:/ /www.msal.gov.ar/archivos/ PARTE\%20RESPIRATORIAS\%20SE\%2034\%20-\%2027\%20 de $\% 20$ agosto $\% 20 \sin \% 20$ cobertura.pdf.

4. Bakir J, Salvay M, Lución F, Gentile A. Factores de riesgo asociados a la infección por virus influenza en pediatría. $6^{\circ}$ Congreso Argentino de Infectología Pediátrica.16 al 19 de abril de 2008. Buenos Aires, 2008. Trabajo No 61.

5. Bakir J, Umido V, Romanin V, Ruttimann R, et al. Impacto del virus influenza en los niños: la realidad en un Hospital Pediátrico. Rev Hosp Niños B Aires 2005;47(214):270-5.

6. Izurieta HS, Thompson WW, Kramarz P, Shay DK, et al. Influenza and the rates of hospitalization for respiratory disease among infants and young children. $N$ Engl J Med 2000;342(4):232-9.

7. World Health Organisation. International Statistical Classification of Diseases and Related Health Problems. 10th Revision. Version for 2010. [Acceso: 19 de diciembre de 2011]. Disponibleen:http://www.who.int/classifications / apps/icd/icd10online/.

8. Nomenclador de Prestaciones de Salud. Gobierno de la Ciudad de Buenos Aires. Argentina. Año 2010.

9. Dean AG, Sullivan KM, Soe MM. OpenEpi: Open Source Epidemiologic Statistics for Public Health, Version 2.3.1. [Acceso: 15 de octubre de 2010]. Disponible en: www.OpenEpi.com, updated 2010/19/09.

10. Lee A, Chuh AA. Facing the threat of Influenza Pandemic: Roles of and implications to general practitioners. $B M C$ Public Health 2010;10(1):661.

11. Koopmans M, Wilbrink B, Conyn M, Natrop G, et al. Transmission of H7N7 avian influenza A virus to human beings during a large outbreak in commercial poultry farms in The Netherlands. Lancet 2004;363(9409):587-93.

12. Nicholson KG, Wood JM, Zambon M. Influenza. Lancet 2003;362(9397):1733-45.

13. Reid A. The effects of the 1918-1919 influenza pandemic on infants and child health in Derbyshire. Med Hist 2005;49(1):29-54.
14. Keren R, Zaoutis TE, Saddlemire S, Luan XQ, Conffin SE. Direct medical cost of influenza-related hospitalizations in children. Pediatrics 2006;118(5):e1321-7.

15. Hall JL, Katz BZ. Cost of influenza hospitalization at a tertiary care children's hospital and its impact on the cost-benefit analysis of the recommendation for universal influenza immunization in children age 6 to 23 months. J Pediatr 2005;147(6):807-11.

16. Ampofo K, Gesteland PH, Bender J, Mills M, et al. Epidemiology, complications, and cost of hospitalization in children with laboratory-confirmed influenza infection. Pediatrics 2006;118(6):2409-17.

17. Peltola V, Ziegler T, Ruuskanen O. Influenza A and B virus infections in children. Clin Infect Dis 2004;4:75-83.

18. Sociedad Argentina de Infectología (SADI), Sociedad Argentina de Pediatría (SAP) (Comité Nacional de Infectología). Documento sobre Infección por virus de influenza A (H1N1). Junio 2009, disponible en: http:/ /www.sap. org.ar/staticfiles/comunicaciones /Documento_GRIPEA_5_7_09.pdf.

19. Ministerio de Salud de la Nación. Plan de Contingencia. 28 de junio de 2009. Disponible en: http:/ / www.msal.gov. ar/archivos/plan-contingencia-2009.pdf.

20. Gentile A, Lucion MF, Castellano V, Dastugue M, et al. Winter season influenza A/H1N1swlvirus epidemiological pattern in a pediatric hospital in Argentina. $47^{\circ}$ Encuentro Anual de la Sociedad Estadounidense de Infectología Pediátrica (IDSA). Filadelfia, EEUU. 29 de octubre-1 de noviembre de 2009.

21. Saldías F, Méndez JI, Ramírez D, Díaz O. Predictive value of history and physical examination for the diagnosis of community-acquired pneumonia in adults. Aliterature review. Rev Med Chil 2007;135(4):517-28. Epub 2007 May 16.

22. Christensen-Szalanski JJ, Bushyhead JB. Physician's use of probabilistic information in a real clinical setting. J Exp Psychol Hum Percept Perform 1981;7:928-35.

23. Noyola D, Clark B, O'Donnell F T, Atmar R, et al. Comparison of a new neuraminidase detection assay with an enzyme immunoassay, immunofluorescence, and culture for rapid detection of Influenza A and B viruses in nasal wash specimens. J Clin Microbiol 2000.Págs.1161-5.

24. EspositoS, Marchisio P, Bosis S, Lambertini L, et al. Clinical and economic impact of influenza vaccination on healthy children aged 2-5 years. Vaccine 24, 2006.Págs.629-35.

25. Principi N, Esposito S, Marchisio P, Gasparini R, et al. Socioeconomic impact of influenza on healthy children and their families. Pediatr Infect Dis J 2003;22(10Suppl):S207-10.

26. Cohen G, Mary D. Nettleman MD. Economic impact of influenza vaccination in preschool children. Pediatrics 2000;106(5):973-976.

\section{Fe de errata}

En el artículo "Infección respiratoria aguda viral en niños menores de 5 años. Estudio epidemiológico en dos centros de Buenos Aires, Argentina", publicado en el volumen 109, número 4, de agosto de 2011, el orden que corresponde de los autores es: Bioq. Débora N. Marcone, Dr. Santiago Vidaurreta, Dr. Alejandro Ellis, Dr. Jorge Ekstrom, Dr. Diego Cukier, Dra. Cristina Videla, Dra. Guadalupe Carballal y Dra. Marcela Echavarría.

Se deja constancia que la Bioq. Marcone y el Dr. Vidaurreta comparten la primera autoría del trabajo. 